\title{
Otimização de metodologia para análise de hidrocarbonetos em sedimentos lagunares
}

Os hidrocarbonetos são listados como alguns dos principais contaminantes ambientais nos dias de hoje e são considerados indicadores geoquímicos em relação aos níveis e fontes de contaminação em ambientes hídricos. Por este motivo existem metodologias padronizadas para a determinação destes contaminantes em sedimentos, entre as mais utilizadas, podemos listar o método de extração por soxhlet e o método de extração sob ultrassom, porém os dois métodos de extração apresentam desvantagens em sua utilização. Neste estudo, foi desenvolvida uma metodologia para a extração, pré concentração e clean up de hidrocarbonetos presentes em sedimento, combinando etapas de extração por solvente sob ultrassom e em soxhlet, seguidas por fracionamento em coluna líquida preparativa e os extratos analisados por cromatografia gasosa acoplada a espectrometria de massas. Para otimização utilizou-se um planejamento experimental $2^{2}$ com ponto central avaliando os tempos de pré sonificação e extração soxhlet. O método que apresentou os melhores resultados, combinou 15 minutos de ultrassom e 4 horas de soxhlet, com recuperações médias de 102,2\% para HPAs deuterados e 100,2\% para o hexadeceno, produzindo uma redução de tempo e volume de solventes, gerando um método reprodutível (RSD\% $<20 \%$ ), rápido e de baixo custo. Os limites de detecção para n-alcanos variaram de 0,9 a 13,9 $\mu \mathrm{g}$ kg-1 e para HPAs de 0,11 a $0,93 \mu \mathrm{g} \mathrm{kg-1.} \mathrm{A} \mathrm{metodologia} \mathrm{foi} \mathrm{aplicada} \mathrm{a} \mathrm{amostras} \mathrm{da} \mathrm{Praia} \mathrm{do} \mathrm{Pontal,} \mathrm{que} \mathrm{faz} \mathrm{parte} \mathrm{da} \mathrm{Lagoa} \mathrm{Mirim,} \mathrm{que} \mathrm{é} \mathrm{um} \mathrm{dos} \mathrm{principais} \mathrm{corpos} \mathrm{hídricos} \mathrm{da} \mathrm{região} \mathrm{Sul} \mathrm{do}$ Brasil e possui grande importância para agricultura, indústria e pecuária. Os níveis de HPAs e n-alcanos indicaram baixos níveis de contaminação e fontes de origem biogênica e pirolítica.

\section{Optimization of methodology for the analysis of hydrocarbons in lagunar sediments}

\begin{abstract}
Hydrocarbons are listed as some of the major environmental contaminants today and are considered geochemical indicators in relation to levels and sources of contamination in water environments. For this reason, there are standardized methodologies for the determination of these contaminants in sediments, among the most used, we can list the method of extraction by soxhlet and the method of extraction under ultrasound, but the two methods of extraction have disadvantages in their use. In this study, a methodology was developed for the extraction, preconcentration and clean up of hydrocarbons present in sediment, combining solvent extraction steps under ultrasound and soxhlet, followed by preparative liquid column fractionation and the extracts analyzed by gas chromatography coupled to mass spectrometry. For optimization, a $2^{2}$ experimental design with central point evaluating the times of pre-sonification and soxhlet extraction was used. The method that presented the best results, combined 15 minutes of ultrasound and 4 hours of soxhlet, with average recoveries of $102.2 \%$ for deuterated PAHs (hydrocarbons polycyclic aromatic) and $100.2 \%$ for hexadecene, producing a reduction of time and volume of solvents, generating a reproducible method (RSD\% $<20 \%$ ), fast and inexpensive. Detection limits for $\mathrm{n}$-alkanes ranged from 0.9 to $13.9 \mu \mathrm{g} \mathrm{kg-1}$ and for PAHs from 0.11 to $0.93 \mu \mathrm{gg}-1$. The methodology was applied to samples of Pontal Beach, which is part of Mirim Lagoon, which is one of the main water bodies in the southern region of Brazil and is of great importance for agriculture, industry and livestock. PAHs and n-alkane levels indicated low levels of contamination and sources of biogenic and pyrolytic origin.
\end{abstract}

Keywords: PAHs; Ahs; N-Alkanes; Soxhlet; Ultrasound; Sample Preparation.

Topic: Desenvolvimento, Sustentabilidade e Meio Ambiente

Reviewed anonymously in the process of blind peer.

\section{Júlia Arduim}

Instituto Federal Sul-Rio-Grandense, Brasil http://lattes.cnpq.br/4547721924522273 juliaarduim@hotmail.com

Helen Cristina dos Santos Hackbart (id) Universidade Federal de Pelotas, Brasil http://lattes.cnpq.br/0709023863824952 http://orcid.org/0000-0001-9176-314X helenhackbart@gmail.com

\section{Maria Alice Farias da Silva Amaral}

Instituto Federal Sul-Rio-Grandense, Brasil http://lattes.cnpq.br/7851108087720691 marryalyce46@icloud.com

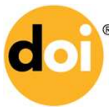

DOI: 10.6008/CBPC2179-6858.2019.003.0015
Received: $12 / 04 / 2019$ Approved: 27/05/2019
Pedro José Sanches Filho (it)

Instituto Federal Sul-Rio-Grandense, Brasil http://lattes.cnpq.br/9785390634457316 http://orcid.org/0000-0002-9852-8940 pjsans@ibest.com.br
Referencing this:

ARDUIM, J.; HACKBART, H. C. S.; AMARAL, M. A. F. S.; SANCHES FILHO, P. J.. Otimização de metodologia para análise de hidrocarbonetos em sedimentos lagunares. Revista Ibero Americana de Ciências Ambientais, v.10, n.3, p.165-178, 2019. DOI: http://doi.org/10.6008/CBPC2179-6858.2019.003.0015 


\section{INTRODUÇÃO}

Os hidrocarbonetos estão listados dentre os principais contaminantes ambientais nos dias de hoje, pois são gerados com facilidade e apresentam uma grande dispersão. Dentro desta função destacam-se os hidrocarbonetos alifáticos (HAs) e os hidrocarbonetos policíclicos aromáticos (HPAs) (HARTZELL et al., 2018). Uma vez no ambiente hídrico, estes compostos são facilmente adsorvidos ao material em suspensão, em função de suas propriedades hidrofóbicas, acumulando-se junto aos sedimentos de rios, lagos e áreas costeiras marinhas. Ocorrem normalmente em misturas complexas provenientes de fontes biogênicas, diagênicas, petrogênicas, pirolíticas. Sua constituição qualitativa e quantitativa pode apresentar informações sobre o nível de impacto em um ambiente hídrico bem como as possíveis fontes de contaminação (SANCHES FILHO et al., 2017).

Para a determinação de hidrocarbonetos em amostras ambientais são necessários métodos de extração, pré concentração e clean up, adequando estes analitos ao método de determinação, como a cromatografia gasosa (BET, 2010). Muitos destes métodos foram padronizados pela United States Environmental Protection Agency (USEPA) pois a extração é considerada uma etapa crítica na determinação de hidrocarbonetos nestes tipos de amostras, uma vez que estes contaminantes hidrofóbicos frequentemente estão fortemente ligados a outros componentes presentes nestas matrizes ambientais. Como técnicas oficiais, podemos citar o método de extração por solvente em soxhlet (EPA 3540C) e extração sob ultrassom (EPA 3550C), porém ambos apresentam desvantagens em sua utilização, o método por soxhlet exige, para uma extração bem-sucedida, de 12 a 36 horas de funcionamento, ainda necessitando de processos de clean-up. A extração sob ultrassom ocorre em menos tempo, porém este método utiliza grandes volumes de solvente, o que expõem o analista a intoxicações pelos períodos de evaporação, tem um custo elevado e é agressivo ao meio ambiente, tendo em vista os resíduos gerados a partir desta extração (USEPA, 1996; USEPA, 2007; CAVALCANTE et al., 2008).

Atualmente tem chamado atenção como métodos alternativos aos métodos oficiais, a extração por fluído supercrítico, extração acelerada por solvente e extração assistida por micro-ondas (PENA et al., 2008), bem como equipamentos que combinam a extração soxhlet com banho ou sonda de ultrassom, porém todas metodologias apresentam desvantagens como alto investimento, alto custo de manutenção e pouca disponibilidade em laboratórios (LUQUE-GARCIA et al., 2004; DJENNI et al., 2013).

Sendo assim, o objetivo deste estudo foi otimizar uma metodologia combinando etapas de pré sonificação e extração em soxhlet, para determinação de hidrocarbonetos (HAs e HPAs) em amostras de sedimento utilizando equipamentos simples, baratos e disponíveis na maioria dos laboratórios, reduzindo tempo de extração e volume de solvente.

\section{MATERIAIS E MÉTODOS}

\section{Otimização da Metodologia}

Para a otimização de metodologia foi realizado o delineamento composto central (DCC) onde foram 
avaliadas duas variáveis independentes: tempo de ultrassom e tempo de soxhlet em relação ao efeito simultâneo nas variáveis dependentes: recuperação de hexadeceno e HPAs deuterados através de um planejamento fatorial completo $2^{2}$ com quatro pontos centrais, totalizando oito experimentos, apresentados na Tabela 1. A análise estatística dos efeitos foi realizada através do programa Statistica 7.1 (STATSOFT, EUA), utilizando um intervalo de confiança de 90 \% (TEÓFILO et al., 2006).

Tabela 1: Variáveis e níveis no planejamento experimental completo $\left(2^{2}\right)$.

\begin{tabular}{|l|l|l|l|}
\hline \multicolumn{1}{|c|}{ Fatores } & \multicolumn{3}{c|}{ Níveis } \\
\hline & -1 & 0 & +1 \\
\hline Tempo de ultrassom (minutos) & 15 & 30 & 45 \\
\hline Tempo de soxhlet (horas) & 4 & 6 & 8 \\
\hline
\end{tabular}

Foram mantidos constantes o volume, a constituição do solvente extrator (100 mL 1:1 hexano/acetona) e a massa da amostra úmida de $20 \mathrm{~g}$. As etapas de pré sonificação foram desenvolvidas em banho ultrassônico (USC 4800A Unique, frequência $40 \mathrm{KHZ}$ ), em temperatura ambiente, em seguida, transferidas para soxhlet.

Para o estudo de recuperação, uma amostra do sedimento coletada na Praia do Pontal (Lagoa Mirim, RS, Brasil) foi fortificada com $200 \mu \mathrm{g} \mathrm{kg-1} \mathrm{(nível} \mathrm{alto)} \mathrm{de} \mathrm{HPAs} \mathrm{deuterados} \mathrm{(acenafteno} \mathrm{-} \mathrm{D10,} \mathrm{fenantreno} \mathrm{-}$ D10 , criseno - D12 e perileno - D12) e 250 mg kg-1 (nível alto) de Hexadeceno (1 - hexadeceno), ambas preparadas em diclorometano (DCM) a partir da diluição dos padrões adquiridos da Supelco (Bellefonte , PA, EUA). O método de extração otimizado foi aplicado a amostras do mesmo ponto, em níveis inferiores de fortificação: $250 \mu \mathrm{g} \mathrm{kg}-1$ para hexadeceno e de $20 \mu \mathrm{g} \mathrm{kg}-1$ para HPAs. Todos os experimentos foram realizados em triplicata para estudo da precisão do método expresso através do desvio padrão relativo em $\%$ (RSD\%).

\section{Fracionamento em coluna cromatográfica líquida preparativa}

Os extratos obtidos foram fracionados em uma coluna de vidro $(21$ x 1,1 cm d.i. e reservatório de 8 x 3,1 cm d.i.) empacotada com $1 \mathrm{~g}$ de $\mathrm{Na}_{2} \mathrm{SO}_{4}$ anidro ativado, agulhas de cobre, 3,2 g de sílica gel 5\% desativada, 1,8 $\mathrm{g}$ de alumina $2 \%$ desativada e $1 \mathrm{~g}$ de $\mathrm{Na}_{2} \mathrm{SO}_{4}$ anidro. Os extratos dispersos em $0,5 \mathrm{~g}$ de sílica foram adicionados no topo de uma coluna. A sequência de solventes para a eluição foi de $20 \mathrm{~mL}$ de hexano, que gerou a fração F1 (HAs), $20 \mathrm{~mL}$ de uma mistura de $\mathrm{DCM} / \mathrm{hexano}(1: 5, \mathrm{v} / \mathrm{v})$ e $20 \mathrm{~mL}$ de uma mistura de DCM/hexano que gerou a fração F2 (HPAs) (UNEP, 1992). Os solventes e reagentes utilizados nesta metodologia foram adquiridos da Merck AS Chemical Industries.

\section{Cromatografia gasosa acoplada a espectrometria de massas}

Uma alíquota de $1 \mu \mathrm{L}$ de cada extrato (em triplicata) foi automaticamente injetada (AOC-20i Shimadzu), em um cromatógrafo gasoso acoplado a um espectrômetro de massas GC/MS-QP2010 Ultra equipada com um sistema de aquisição de dados GC Solution Shimadzu. Para a análise de HPAs o programa de temperatura foi otimizado com uma solução padrão de $4 \mathrm{mg} \mathrm{L}^{-1}$ contendo os 16 HPAs listados como prioritários pela USEPA, adquiridos da Supelco (Bellefonte, PA, EUA) e a mistura dos 4 HPAs deuterados, 
inicialmente injetados em modo SCAN e Splitless para a otimização das janelas de tempo e definição dos íons a serem monitorados, ficando definida as condições expressas na Tabela 2. Para a determinação de n-alcanos o programa de temperatura foi ajustado a partir de uma solução $5 \mathrm{mg} \mathrm{L}^{-1}$, em DCM composta por n-alcanos de C8-C40 obtidos a partir da diluição dos padrões de C8-C20 e C21 a C40 adquiridas da Sigma Aldrich (Steinheim, Suíça).

Tabela 2: Condições cromatográficas para a análise em GC/MS.

\begin{tabular}{|c|c|}
\hline Parâmetro & Especificação \\
\hline Coluna & $\begin{array}{l}\text { RTx-5ms coluna ( } 30 \mathrm{~m} \times 0,25 \mathrm{~mm} \text { d.i. } \times 0,25 \mu \mathrm{m} \text { espessura de filme (J \& W Scientific, } \\
\text { Folson, CA, USA) }\end{array}$ \\
\hline Modo de Injeção & Splitless \\
\hline Temperatura do injetor & $280^{\circ} \mathrm{C}$ \\
\hline Temperatura da interface & $200^{\circ} \mathrm{C}$ \\
\hline Ionização & Eletron-Impacto (EI) em $70 \mathrm{eV}$ \\
\hline Gás de transporte/fluxo & Hélio $\left(1,0 \mathrm{~mL} \mathrm{~min}^{-1}\right)$ \\
\hline Temperatura do forno (F2 - HPAs) & Temperatura inicial $60^{\circ} \mathrm{C}(5 \mathrm{~min}) 8^{\circ} \mathrm{C} \min ^{-1}, 200^{\circ} \mathrm{C},(0 \mathrm{~min}), 5^{\circ} \mathrm{C} \min 300{ }^{\circ} \mathrm{C}{ }^{1}(15 \mathrm{~min})$. \\
\hline $\begin{array}{l}\text { Temperatura do forno (F1 - } \mathrm{n} \\
\text { alcanos) }\end{array}$ & Temperatura inicial $60^{\circ} \mathrm{C}(10 \mathrm{~min}) 5^{\circ} \mathrm{C} \mathrm{min}^{-1}, 200^{\circ} \mathrm{C},(5 \mathrm{~min}), 10^{\circ} \mathrm{C} \min 300{ }^{\circ} \mathrm{C}(35 \mathrm{~min})$. \\
\hline $\begin{array}{l}\text { Janelas e Íons Monitorados (modo } \\
\text { SIM) } \\
\text { HPAs }\end{array}$ & $\begin{array}{l}\text { Janela 1: 5-18 minutos, } \mathrm{m} / \mathrm{z}: 128,136 ; \\
\text { Janela 2: } 18-36 \text { minutos, } \mathrm{m} / \mathrm{z}: 152,154,164,166,178,202,228,240 \text {, } \\
\text { Janela 3: } 33-55 \text { minutos, } \mathrm{m} / \mathrm{z}: 252,264,276,278\end{array}$ \\
\hline
\end{tabular}

Todos os procedimentos foram acompanhados com análise de brancos, para avaliar possíveis interferências de contaminantes do ambiente laboratorial bem como reagentes. Afim de verificar o desempenho dos métodos cromatográficos, a linearidade foi estudada a partir coeficiente de correlação $\left(r^{2}\right)$ gerados a partir das curvas de calibração de $0,5-5 \mathrm{mg} \mathrm{L}^{-1}$ para $\mathrm{n}$-alcanos e 20-200 $\mathrm{g} \mathrm{L} \mathrm{L}^{-1}$ para HPAs como sugerido pela EPA 5035A (2002).

A precisão dos métodos cromatográficos foi desenvolvida repetindo 11 vezes a análise do padrão de $50 \mu \mathrm{g} \mathrm{L}^{-1}$ para HPAs e do padrão de $4 \mathrm{mg} \mathrm{L}^{-1}$ para $\mathrm{n}$-alcanos. As exatidões foram analisadas por meio do erro relativo em relação aos mesmos padrões. O limite de deteç̧ão (LD) foi calculado utilizando-se três vezes o desvio padrão do sinal do branco, enquanto que o limite de quantificação (LQ) foi calculado dez vezes o desvio padrão do sinal do branco, ambos divididos pelo coeficiente angular. Para o cálculo do LD e LQ do método se considerou uma massa de $20 \mathrm{~g}$ de amostra com uma recuperação de 100\% de cada analito.

Com o objetivo de identificar a origem dos HPAs e diferenciar as fontes petrogênica e fonte pirolítica, foram calculadas as razões diagnósticas para as amostras de sedimento. As relações Fen/Ant, Ant/(Ant+Fen), $\mathrm{Flu/(Flu} \mathrm{+} \mathrm{Pir),} \mathrm{Flu/Pir} \mathrm{estão} \mathrm{associadas} \mathrm{a} \mathrm{entradas} \mathrm{pirogênicas} \mathrm{ou} \mathrm{petrogênicas.} \mathrm{A} \mathrm{razão} \mathrm{Flu/(Flu+Pir)} \mathrm{é} \mathrm{um}$ indicadore de combustão de combustíveis fósseis, de grama, madeira ou carvão (HASSAN et al., 2018). Para n-alcanos o índice de preferência de carbono (IPC) é um meio numérico de representar a predominância ímpar/par em hidrocarbonetos alifáticos em uma faixa particular de carbono (ASSUNÇÃO et al., 2018) e pode ser calculada da seguinte forma:

$$
I P C=\frac{1}{2}\left[\frac{\mathrm{C} 25+\mathrm{C} 27+\mathrm{C} 29+\mathrm{C} 31+\mathrm{C} 33}{\mathrm{C} 24+\mathrm{C} 26+\mathrm{C} 28+\mathrm{C} 30+\mathrm{C} 32}\right]+\left[\frac{\mathrm{C} 25+\mathrm{C} 27+\mathrm{C} 29+\mathrm{C} 31+\mathrm{C} 33}{\mathrm{C} 26+\mathrm{C} 28+\mathrm{C} 30+\mathrm{C} 32+\mathrm{C} 34}\right]
$$

A razão terrestre/aquática (RTA) é a relação entre as concentrações de n-alcanos de cadeia longa (nC27 + nC29 + nC31) sobre os n-alcanos de cadeia curta (nC15 + nC17 + nC19) e avalia a prevalência de fontes aquáticas ou terrestres. Além destes resultados, as MCNR foram determinadas através de um fator 
de resposta médio, calculado a partir dos padrões de $n$-alcanos. A razão $M C N R / \Sigma n$-alcanos indica uma contaminação por combustível residual crônica ou recente (TOLOSA et al., 2004).

\section{Amostragem e caracterização preliminar}

Para aplicação da metodologia otimizada à amostras reais, foram utilizados os sedimentos

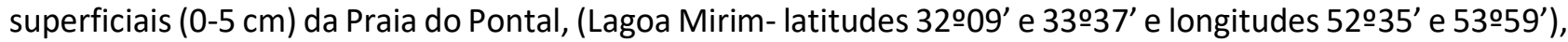
coletados em três pontos (Agosto de 2018) com uma draga de aço inoxidável do tipo 'Van Veen', como descrito por Mozeto (2007). A amostra utilizada para a otimização de metodologia foi coletada no ponto 2 . A Figura 1 apresenta os pontos de coleta, bem como as georreferencias determinadas pelo GPS (GARMINETREX 10).
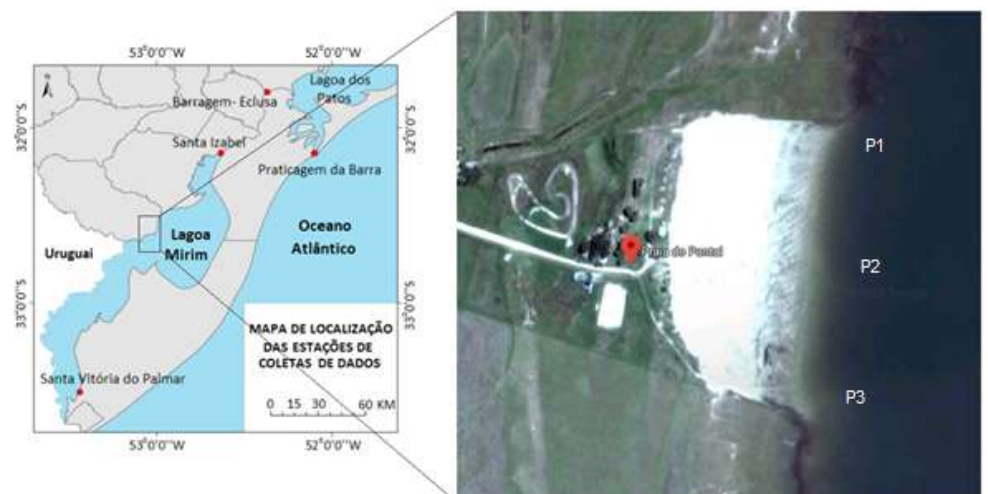

Figura 1: Localização geográfica dos pontos de amostragem na Praia do Pontal/RS, Brasil.

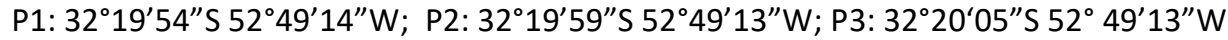

Fonte: Adaptado de Oliveira et al. (2015).

O material coletado foi retirado e armazenado em potes de vidro previamente descontaminados. Após a coleta as amostras foram devidamente transportadas e armazenadas a $4{ }^{\circ} \mathrm{C}$. Parte das amostras foi reservada para determinação de umidade, matéria orgânica, granulometria e pH. Todas análises físicoquímicas foram realizadas em triplicata.

A determinação da matéria orgânica no sedimento foi realizada por perdas de voláteis, a uma temperatura de $550^{\circ} \mathrm{C}$, por um período de 4 horas (APHA, 2005). A análise granulométrica foi realizada pelo método descrito por Suguio (1973) que consiste em peneirar o sedimento utilizando a escala de Wertworth, por tamanhos dos grãos: grão $(2 \mathrm{~mm})$, areia muito grossa $(1 \mathrm{~mm})$, areia grossa (500 $\mu \mathrm{m})$, areia média (250 $\mu \mathrm{m})$, areia fina $(125 \mu \mathrm{m})$, areia muito fina $(63 \mu \mathrm{m})$, fundo $(<63 \mu \mathrm{m})$. A umidade foi avaliada com cerca de 10 $\mathrm{g}$ de amostra em estufa com temperatura a $105{ }^{\circ} \mathrm{C}$, durante 24 horas (APHA, 2005). $\mathrm{O} \mathrm{pH}$ do sedimento foi determinado em suspensão utilizando a proporção 1:2 (sedimento/água do mesmo ponto) como empregada por Yoon et al. (2006).

\section{RESULTADOS E DISCUSSÃO}

\section{Otimização de metodologia}

Neste estudo foram avaliados o efeito das variáveis: tempo de ultrassom $\left(\mathrm{x}_{1}\right)$ e tempo de soxhlet $\left(\mathrm{x}_{2}\right)$, na recuperação de hexadeceno e HPAs deuterados em sedimentos previamente fortificados, esses 
resultados estão apresentados na Tabela 3, no qual a recuperação está expressa em porcentagem.

Tabela 3: Variáveis e recuperação de hexadeceno e HPAs para o planejamento fatorial completo $2^{2}$.

\begin{tabular}{|l|l|l|l|l|}
\hline Ensaio & Tempo de Ultrassom (min) & Tempo de Soxhlet $(\mathrm{h})$ & Hexadeceno (\%) & HPAs Deuterados (\%) \\
\hline 1 & $-1(15)$ & $-1(4)$ & 116,4 & 107,8 \\
\hline 2 & $1(45)$ & $1(8)$ & 45,1 & 24,4 \\
\hline 3 & $-1(15)$ & $1(8)$ & 53,7 & 40,2 \\
\hline 4 & $1(45)$ & $-1(4)$ & 133,4 & 56,5 \\
\hline 5 & $0(30)$ & $0(6)$ & 120,3 & 77,8 \\
\hline 6 & $0(30)$ & $0(6)$ & 115,3 & 69,5 \\
\hline 7 & $0(30)$ & $0(6)$ & 108,6 & 71,2 \\
\hline 8 & $0(30)$ & $0(6)$ & 113,1 & 70,1 \\
\hline
\end{tabular}

Os resultados de recuperação dos experimentos 1 a 4 corresponde a médias de duas extrações.

A análise estatística mostrou que o tipo de extração influenciou significativamente na recuperação tanto do hexadeceno quanto dos HPAs deuterados, conforme mostra a Tabela 4, onde é possível observar que apenas a variável tempo de ultrassom, para o resultado de recuperação do hexadeceno, não foi significativa com valor de $p>0,10$, no entanto, o tempo de soxhlet apresentou um efeito negativo, ou seja, o menor tempo estudado resultou em uma maior recuperação de hexadeceno, assim como a interação entre o tempo de ultrassom e o tempo de soxhlet. Para a recuperação dos HPAs deuterados, todas as variáveis foram significativas para o intervalo de confiança de $90 \%$. Tanto o tempo de ultrassom, quanto o tempo de soxhlet apresentaram efeitos negativos, ou seja, o menor tempo utilizado aumentou a recuperação destes compostos.

Tabela 4: Efeitos e coeficientes para as diferentes extrações.

\begin{tabular}{|l|l|l|l|l|l|l|}
\hline & \multicolumn{2}{|l|}{ Hexadeceno } & \multicolumn{2}{l|}{ HPAs Deuterados } \\
\hline & Efeito & Desvio Padrão & Significância $(p)$ & Efeito & Desvio Padrão & Significância (p) \\
\hline Média & 101,010 & 1,6976 & 0,000010 & 64,626 & 1,3805 & 0,000021 \\
\hline Ultrassom (minutos) & 4,190 & 4,8016 & 0,447093 & $-33,590$ & 3,9047 & 0,003303 \\
\hline Soxhlet (horas) & $-75,510$ & 4,8016 & 0,000559 & $-49,870$ & 3,9047 & 0,001036 \\
\hline Ultrassom versus Soxhlet & $-12,810$ & 4,8016 & 0,075830 & 17,770 & 3,9047 & 0,019880 \\
\hline
\end{tabular}

$\mathrm{p}=$ nível de significância de $90 \%$.

Os coeficientes de regressão, apresentados na Tabela 5, mostram um modelo possível de validar, em função das variáveis estudadas, por meio da análise de variância, onde se observou que estes foram preditivos e significativos.

Tabela 5: Coeficientes de regressão para as variáveis significativas para recuperação de hexadeceno e HPAs deuterados.

\begin{tabular}{|l|l|l|l|l|l|l|}
\hline \multicolumn{2}{|l|}{ Hexadeceno } & \multicolumn{3}{l|}{ HPAs deuterados } \\
\hline & Coef. Reg. & Desvio padrão & $\begin{array}{l}\text { Signif. } \\
(p)\end{array}$ & Coef. Reg. & Desvio padrão & Signif. (p) \\
\hline Média & 101,010 & 1,6976 & 0,000010 & 64,626 & 1,3805 & 0,000021 \\
\hline Ultrassom (minutos) & & & & $-16,795$ & 1,9523 & 0,003303 \\
\hline Soxhlet (horas) & $-37,755$ & 2,4008 & 0,000559 & $-24,935$ & 1,9523 & 0,001036 \\
\hline Ultrassom versus Soxhlet & $-6,405$ & 2,4008 & 0,075830 & 8,885 & 1,9523 & 0,019880 \\
\hline
\end{tabular}

$\mathrm{p}=$ nível de significância de $90 \%$.

É possível pelos coeficientes de regressão gerar os modelos, apresentados nas equações 1 e 2, que prediz a resposta das variáveis: hexadeceno $\left(Y_{1}\right)$ e HPAs deuterados $\left(Y_{2}\right)$ em função das variáveis tempo de ultrassom e soxhlet.

$$
Y_{1}=101,010-37,755 x_{2}-6,405 x_{1} x_{2}
$$

equação 1 


$$
Y_{2}=64,626-16,795 x_{1}-24,935 x_{2}+8,885 x_{1} x_{2}
$$

Onde: $\mathrm{x}_{1}=$ tempo de ultrassom (minutos) e $\mathrm{x}_{2}=$ tempo de soxhlet (horas) Os resultados preditivos e significativos são comprovados a partir do teste $\mathrm{F}$, no qual o $\mathrm{F}$ calculado foi maior que o $\mathrm{F}$ tabelado para as duas variáveis estudadas, tempo de ultrassom e tempo de soxhlet, estes valores estão apresentados na Tabela 6. Assim, a partir dos modelos, equações 1 e 2, é possível gerar a superfície de resposta para a variável HPAs deuterados, a qual, apresentou todas as variáveis significativas, (Figura 2).

Tabela 6: Dados de ANOVA para o planejamento experimental $2^{2}$.

\begin{tabular}{|c|c|c|c|c|c|c|}
\hline \multicolumn{7}{|l|}{ Hexadeceno } \\
\hline Fonte de variação & Soma quadrática & Grau de liberdade & Média quadrática & Teste $\mathrm{F}$ & Fcalc/Ftab & $\mathrm{R}^{2}$ \\
\hline Regressão & 5865,856 & 2 & 2932,928 & 9,026 & 2,39 & 78,31 \\
\hline Resíduo & 1624,63 & 5 & 324,926 & & & \\
\hline Falta de ajuste & 1555,462 & & & & & \\
\hline Erro puro & 69,168 & & & & & \\
\hline Total & 7490,486 & 7 & & & & \\
\hline \multicolumn{7}{|l|}{ HPAs Deuterados } \\
\hline Fonte de variação & Soma quadrática & Grau de liberdade & Média quadrática & Teste $\mathrm{F}$ & Fcalc/Ftab & $\mathrm{R}^{2}$ \\
\hline Regressão & 2802,79 & 2 & 1401,395 & 4,349 & 1,15 & 63,5 \\
\hline Resíduo & 1611,075 & 5 & 322,215 & & & \\
\hline Falta de ajuste & 1565,333 & & & & & \\
\hline Erro puro & 45,742 & & & & & \\
\hline Total & 4413,865 & 7 & & & & \\
\hline
\end{tabular}

Fcal: F calculado; Ftab: F tabelado2, 5; 0,10 =3,78; $R^{2}$ : \% de variação explicada.

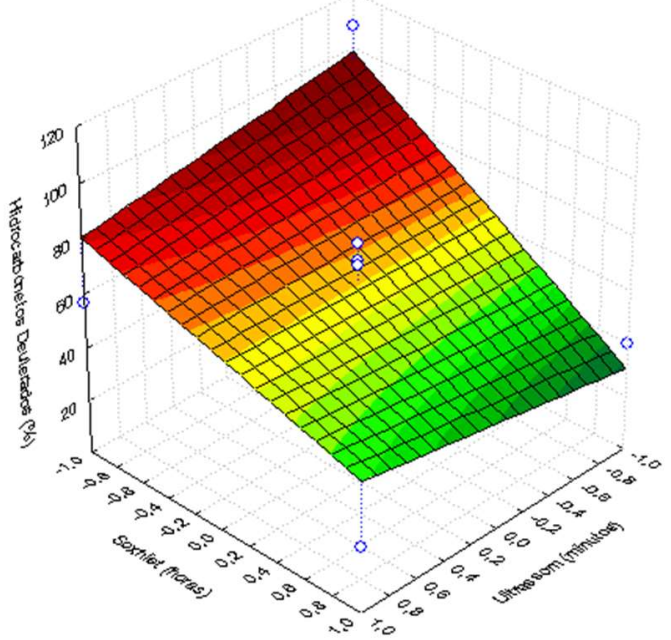

Figura 2: Superfície de resposta e curva de contorno para a recuperação dos HPAs deuterados.

A Figura 2 ilustra que a maior recuperação de HPAs deuterados ocorre quando é utilizado um menor tempo de ultrassom quanto de soxhlet, ou seja, quando é utilizado 15 minutos de ultrassom ou 4 horas de soxhlet (ensaio 1). Considerando os ensaios 2 e 4, onde o tempo de ultrassom permanece fixo em 45 minutos, observa-se uma redução nas recuperações tanto para os HPAs quanto para o hexadeceno, com o aumento de 4 para 8 horas em extração por soxhlet, o que pode indicar perdas durante as etapas de refluxo, tal comportamento é confirmado quando observa-se os resultados dos ensaio 1 e 3 , porém com um menor tempo de pré sonificação.

Os valores de recuperação no ponto central, foram satisfatórios para o hexadeceno e apresentaram uma leve redução para os HPAs deuterados, mesmo assim dentro de uma faixa aceitável. De acordo com as 
normas da União Européia (UE) regulamento 836/2011, valores de recuperação entre $50-120 \%$ são aceitáveis para esta matriz (COMMISSION REGULATION, 2011).

A repetição, em triplicata, do experimento 1 com amostras em níveis inferiores de fortificação geraram recuperações médias de $63,1 \% \pm 12,3 \%$ para HPAs deuterados e $67,0 \% \pm 8,9 \%$ para o hexadececeno. O valores continuam em acordo com as exigências da UE com precisões aceitáveis expressas através do desvio padrão relativo (\%) inferiores a $20 \%$, segundo Brito et al. (2003).

Sun et al. (2018) apresentou recuperações para HPAs deuterados de $74 \% \pm 9 \%$, e $95 \% \pm 6 \%$ utilizando extração sob ultrassom (EPA 3550C) para seus estudos. Qu et al. (2018), usando a metodologia por soxhlet (EPA 3540C), encontrou valores de recuperações para HPAs deuterados que variaram de $67,4 \%$ a $96,2 \%$, os quais equivalentes aos resultados obtidos neste trabalho. $O$ mesmo se aplica para as recuperações de hexadeceno, pois Lourenço et al. (2017) utilizando ultrassom, encontrou valores entre $65 \%$ a $105 \%$ e Bet. (2010), utilizando a extração por soxhlet, apresentou resultados que variam de $61,6 \%$ a $98,0 \%$, assim percebe-se que a metodologia otimizada em acordo com os métodos tradionalmente usados e com o recomendado pela UE 836/2011.

\section{Figuras de mérito para os métodos cromatográficos}

As figuras de mérito para os métodos cromatográficos propostos para análise de hidrocarbonetos estão apresentadas na Tabela 7 Os coeficientes de correlação obtidos tanto para HPAs quanto para os nalcanos foram superiores a 0,98 , situaram-se acima do preconizado $(>0,9)$ como adequado na validação de métodos pelo INMETRO. Os métodos apresentaram exatidão e precisão aceitáveis para a maioria dos analitos, com ER\% entre 0,1 e 2,2\% e RSDs entre 4,8 a 18,1\% para HPAs e ER\% entre $-0,1$ a 10,5\% e RSD\% e 0,1 a 9,8\% para $n$ alcanos. Os HPAs foram avaliados na faixa de $\mu \mathrm{g}$ L-1 que admite RSD até $20 \%$ enquanto os n-alcanos foram avaliados na faixa de mg L-1 admitindo RSDs na ordem de 5\%, apenas os $\mathrm{n}$-alcanos superiores a C37 apresentaram RSDs superiores a este limite (INMETRO, 2003).

Os limites de deteç̧ão para análise de HPAs variaram de 0,004 a 0,067 $\mu \mathrm{g}$ kg-1, em uma faixa mais baixa que os obtidos por Luz et al. (2010), que apresentou variações de LDs de 0,11 a 0,93 $\mu \mathrm{g}$ kg-1. Os LQs variaram entre 0,014 a 0,224 $\mu \mathrm{g} \mathrm{kg}-1$, em acordo com outros estudos, como Souza et al. (2018), que determinaram um valor médio de $0,2 \mu \mathrm{g}$ kg-1.

Em pesquisa realizada por Sanches Filho et al. (2013), na determinação de n-alcanos em sedimento lagunares (Colônia Z3, Lagoa dos Patos/RS/Brasil), foram obtidos resultados para LD e LQ entre 0,2 a 3,8 $\mu \mathrm{g}$ kg-1 e 0,4 a 5,1 ㅆ kg-1, respectivamente, tais concentrações são maiores quando comparadas com os dados encontrados neste estudo (Tabela 7), que apresenta uma variação de 0,02 a 0,28 $\mu \mathrm{g} \mathrm{kg-1} \mathrm{para} \mathrm{LD} \mathrm{e} \mathrm{0,06} \mathrm{a}$ $0,93 \mu \mathrm{g} \mathrm{kg-1}$ para LQ.

O coeficiente angular (a), reflete a sensibilidade do método analítico, tanto para os HPAs quanto nalcanos e observou-se uma queda na sensibilidade com o aumento do peso molecular. Este comportamento pode estar relacionado a redução do fator de resposta (Figura 3a, 3b, 3c), que também prejudica a exatidão e precisão dos métodos. 
Tabela 7: Figuras de mérito para método cromatográfico para análise de HPAs e n-alcanos.

\begin{tabular}{|c|c|c|c|c|c|c|c|c|c|c|}
\hline $\mathrm{N}^{\circ} /$ pico & HPAs & $r^{2}$ & $\mathrm{a}$ & $\mathrm{b}$ & ER\% & RSD $\%$ & $\begin{array}{l}\text { LD } \\
\mu \mathrm{g} \mathrm{kg}^{-1}\end{array}$ & $\begin{array}{l}\text { LQ } \\
\mu \mathrm{g} \mathrm{kg}^{-1}\end{array}$ & $\begin{array}{l}\text { LD } \\
\mu \mathrm{g} \mathrm{kg}^{-1}\end{array}$ & $\begin{array}{l}\text { LQ } \\
\mu \mathrm{kg}^{-1}\end{array}$ \\
\hline 1 & Naftaleno & 0,996 & 1732,6 & 9292,9 & 0,2 & 4,8 & 0,1 & 0,2 & 0,004 & 0,014 \\
\hline 2 & Acenaftileno & 0,999 & 947,4 & 6846,1 & 0,3 & 7,1 & 0,1 & 0,3 & 0,007 & 0,023 \\
\hline 3 & Acenafteno & 0,998 & 1099,6 & 6846,1 & 2,5 & 12,1 & 0,1 & 0,3 & 0,007 & 0,022 \\
\hline 4 & Fluoreno & 0,995 & 478,6 & 3122,9 & 0,6 & 5,6 & 0,2 & 0,7 & 0,014 & 0,047 \\
\hline 5 & Fenantreno & 0,997 & 428,9 & 4672,1 & 0,1 & 7,1 & 0,2 & 0,6 & 0,014 & 0,046 \\
\hline 6 & Antraceno & 0,999 & 349,5 & 3949,0 & 0,3 & 6,5 & 0,2 & 0,8 & 0,017 & 0,057 \\
\hline 7 & Fluoranteno & 0,999 & 438,8 & 5428,4 & 0,4 & 7,9 & 0,2 & 0,6 & 0,013 & 0,045 \\
\hline 8 & Pireno & 0,999 & 526,4 & 5227,3 & 0,2 & 12,9 & 0,2 & 0,5 & 0,011 & 0,037 \\
\hline 9 & Benzo(a)Antraceno & 0,999 & 254,7 & 3232,9 & 0,1 & 18,1 & 0,3 & 1,0 & 0,021 & 0,069 \\
\hline 10 & Criseno & 0,999 & 269,9 & 3367,9 & 0,6 & 10,7 & 0,3 & 0,9 & 0,02 & 0,065 \\
\hline 11 & Benzo(k)Fluoranteno & 0,998 & 222,7 & 2042,5 & 0,8 & 14,6 & 0,5 & 1,6 & 0,035 & 0,117 \\
\hline 12 & Benzo(b)Fluoranteno & 0,996 & 266,8 & 2486,2 & 1,4 & 13,8 & 0,4 & 1,4 & 0,029 & 0,098 \\
\hline 13 & Benzo(a)Pireno & 0,998 & 175,0 & 2959,6 & 0,9 & 10,9 & 0,6 & 2,1 & 0,045 & 0,149 \\
\hline 14 & Benzo(g,h,i)Perileno & 0,995 & 121,2 & 866,6 & 1,3 & 14,3 & 0,8 & 2,5 & 0,054 & 0,18 \\
\hline 15 & Dibenzo(a,h)Antraceno & 0,997 & 97,5 & 225,3 & 1,2 & 15,0 & 0,9 & 3,1 & 0,067 & 0,224 \\
\hline \multirow[t]{2}{*}{16} & Indeno(1,2,3-cd)Pireno & 0,991 & 129,9 & 1047,5 & 2,2 & 14,0 & 0,6 & 1,9 & 0,041 & 0,136 \\
\hline & $\mathrm{N}$-alcanos & & & & & & $\begin{array}{l}\mathrm{LD} \\
\mathrm{mg} \mathrm{L}^{-1}\end{array}$ & $\underset{1}{\mathrm{LQ}} \mathrm{mg} \mathrm{\textrm {L } ^ { - }}$ & $\begin{array}{l}\mathrm{LD} \\
\mathrm{mg} \mathrm{kg}^{-1}\end{array}$ & $\begin{array}{l}\mathrm{LQ} \\
\mathrm{mg} \mathrm{kg}^{-1}\end{array}$ \\
\hline C9 & Nonano & 0,982 & 398093 & -17619 & 10,5 & 3,3 & 0,9 & 3,1 & 0,02 & 0,06 \\
\hline $\mathrm{C} 10$ & Decano & 0,984 & 270819 & -96537 & 7,7 & 1,8 & 1,4 & 4,6 & 0,03 & 0,09 \\
\hline C11 & Undecano & 0,988 & 219751 & -79230 & 4,1 & 0,1 & 1,7 & 5,7 & 0,03 & 0,11 \\
\hline $\mathrm{C} 12$ & Dodecano & 0,987 & 213123 & -94489 & 2,3 & 0,9 & 1,8 & 5,8 & 0,04 & 0,12 \\
\hline $\mathrm{C} 13$ & Tridecano & 0,994 & 203842 & -97901 & 1,0 & 1,2 & 1,8 & 6,1 & 0,04 & 0,12 \\
\hline C14 & Tetradecano & 0,990 & 180282 & -74533 & 1,9 & 0,8 & 2,1 & 6,9 & 0,04 & 0,14 \\
\hline C15 & Pentadecano & 0,995 & 184178 & -65309 & 3,0 & 1,4 & 2,0 & 6,8 & 0,04 & 0,14 \\
\hline C16 & Hexadecano & 0,987 & 187840 & -86901 & 4,0 & 0,8 & 2,0 & 6,6 & 0,04 & 0,13 \\
\hline $\mathrm{C} 17$ & Heptadecano & 0,990 & 193218 & -89607 & 4,2 & 1,8 & 1,9 & 6,4 & 0,04 & 0,13 \\
\hline $\mathrm{C} 18$ & Octadecano & 0,991 & 207847 & -84859 & 3,5 & 1,6 & 1,8 & 6,0 & 0,04 & 0,12 \\
\hline C19 & Nonadecano & 0,989 & 192507 & -96954 & 1,6 & 1,6 & 1,9 & 6,5 & 0,04 & 0,13 \\
\hline $\mathrm{C} 20$ & Eicosano & 0,989 & 187075 & -92973 & 1,9 & 0,9 & 2,0 & 6,7 & 0,04 & 0,13 \\
\hline $\mathrm{C} 21$ & Heneicosano & 0,986 & 223906 & -11896 & 2,3 & 1,3 & 1,7 & 5,6 & 0,03 & 0,11 \\
\hline $\mathrm{C} 22$ & Docosano & 0,989 & 211339 & -10568 & $-0,1$ & 1,9 & 1,8 & 5,9 & 0,04 & 0,12 \\
\hline $\mathrm{C} 23$ & Tricosano & 0,989 & 211433 & -11888 & 2,5 & 0,3 & 1,8 & 5,9 & 0,04 & 0,12 \\
\hline $\mathrm{C} 24$ & Tetracosano & 0,992 & 213815 & -12287 & $-1,0$ & 1,3 & 1,7 & 5,8 & 0,03 & 0,12 \\
\hline $\mathrm{C} 25$ & Pentacosano & 0,992 & 214914 & -11796 & 4,2 & 1,3 & 1,7 & 5,8 & 0,03 & 0,12 \\
\hline $\mathrm{C} 26$ & Hexacosano & 0,993 & 211536 & -10227 & 2,7 & 0,3 & 1,8 & 5,9 & 0,04 & 0,12 \\
\hline $\mathrm{C} 27$ & Heptacosano & 0,987 & 207241 & -12348 & 3,6 & 1,0 & 1,8 & 6,0 & 0,04 & 0,12 \\
\hline $\mathrm{C} 28$ & Octacosano & 0,994 & 197110 & -95354 & $-0,3$ & 1,5 & 1,9 & 6,3 & 0,04 & 0,13 \\
\hline C29 & Nonacosano & 0,991 & 166925 & -85408 & 3,5 & 1,1 & 2,2 & 7,5 & 0,04 & 0,15 \\
\hline C30 & Triacontano & 0,991 & 142747 & -80628 & 0,5 & 0,4 & 2,6 & 8,7 & 0,05 & 0,17 \\
\hline C31 & Untriacontano & 0,995 & 122103 & -68544 & 1,5 & 2,0 & 3,1 & 10,2 & 0,06 & 0,20 \\
\hline $\mathrm{C} 32$ & Dotriacontano & 0,994 & 91946 & -34973 & $-0,2$ & 1,5 & 4,1 & 13,6 & 0,08 & 0,27 \\
\hline C33 & Tritriacontano & 0,990 & 77413 & -18877 & $-2,4$ & 1,4 & 4,8 & 16,1 & 0,10 & 0,32 \\
\hline C34 & Tetratriacontano & 0,988 & 80438 & -41219 & $-4,4$ & 0,1 & 4,6 & 15,5 & 0,09 & 0,31 \\
\hline $\mathrm{C} 35$ & Pentatricontano & 0,991 & 50538 & 29179 & 5,8 & 2,3 & 7,4 & 24,7 & 0,15 & 0,49 \\
\hline C36 & Hexatriacontano & 0,988 & 28342 & 11990 & 8,2 & 4,2 & 13,2 & 44,0 & 0,26 & 0,88 \\
\hline C37 & Heptatriacontano & 0,986 & 26822 & -13840 & $-5,6$ & 7,9 & 13,9 & 46,5 & 0,28 & 0,93 \\
\hline $\mathrm{C} 38$ & Octatriacontano & 0,994 & 147462 & -26411 & 5,7 & 9,2 & 2,5 & 8,5 & 0,05 & 0,17 \\
\hline C39 & Nonatriacontano & 0,990 & 95343 & -16030 & 7,4 & 13,2 & 3,9 & 13,1 & 0,08 & 0,26 \\
\hline $\mathrm{C} 40$ & Tetratriacontano & 0,989 & 91214 & -15015 & 3,5 & 9,8 & 4,1 & 13,7 & 0,08 & 0,27 \\
\hline
\end{tabular}

$\mathrm{Y}=\mathrm{aX}+\mathrm{b}=\mathrm{a}$ : coeficiente angular; $\mathrm{b}$ : coeficiente linear; $r 2$ : coeficiente correlação linear; ER: erro relativo; RSD: desvio padrão relativo; LD: limite de detecção; LQ: limite de quantificação;

Para o HPAs os maiores erros relativos e maiores RSD foram encontrado para compostos com peso molecular acima de 228 u já para os $n$ alcanos os maiores erros relativos e maiores RSD foram determinados para os compostos de menor e maior cadeia carbônica, como observado para o nonano, decano, hexatriacontano e nonatriacontano, este comportamento pode ser explicado pela redução e assimetria dos picos C9 e C10 para n-alcanos e 14, 15 e 16 para HPAs como observado na Figura 3c e 3d. 

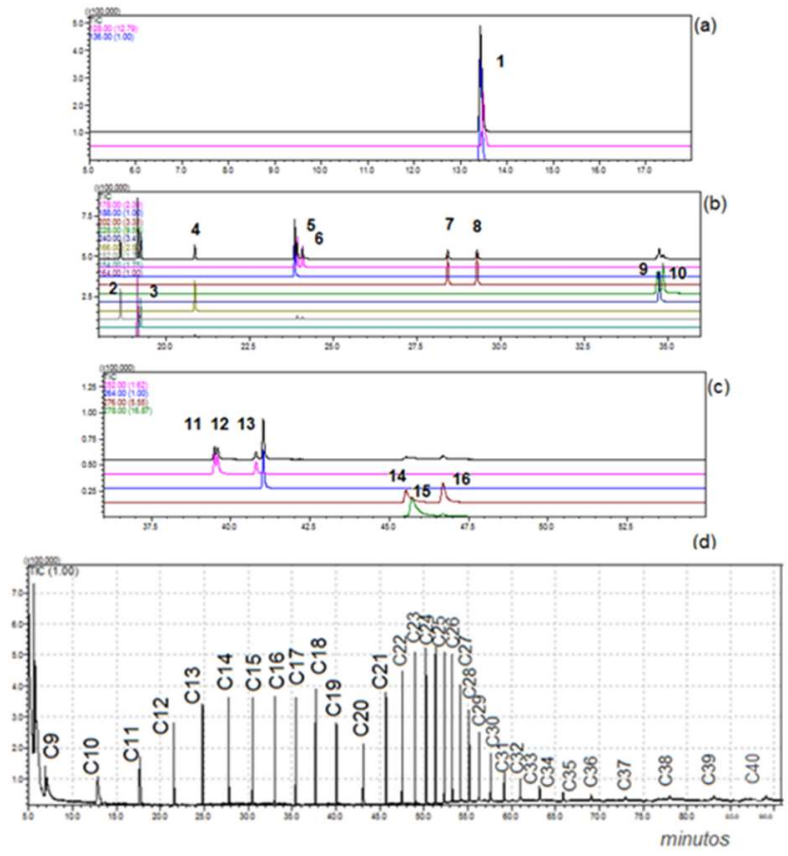

Figura 3: Cromatogramas apresentando as janelas de tempo de retenção para os 16 HPAs prioritários. Janela 1 (a); Janela 2 (b); Janela 3 (c); padrão de n-alcanos, utilizado para otimização de metodologia e demonstrando a perda de sensibilidade analítica pelos picos de C9, C10 e C35 a C40 (d).

\section{Caracterização preliminar}

A Tabela 8, apresenta os resultados de matéria orgânica (MO), pH e umidade para as três amostras analisadas. Nesse estudo foi possível verificar a baixa concentração de matéria orgânica no sedimento, entre 0,5 a 1,7\%, valores que foram similares aos resultados obtidos por Santos et al. (2003), que obteve 0,21 a 2,4\% de matéria orgânica em sedimentos da lagoa mirim, o que mostra um padrão de baixas concentrações de MO nos sedimentos da região, indicando sedimentos inorgânicos.

Os sedimentos possuem uma granulometria homogênea, com predominância de grãos com 0,125 e 0,63 mm. As amostras P2 e P3 tiveram uma porcentagem de, respectivamente, 58,0\% e 56,9\% de grãos com diâmetro de 0,125 mm, já a amostra P1 apresentou $62,0 \%$ de sua composição 0,63 mm, conferindo para as amostras, as características de areia fina e muito fina, de acordo com a escala Wertworth.

Entre as amostras houve baixa variação nos níveis de umidade, que foi de $24,8 \%$ a $27,4 \%$, já os resultados de pH mantiveram-se na zona do neutro, entre 6,5 a 7, esses resultados são similares aos obtidos por Santos et al. (2003), que encontrou um valor médio para pH dos sedimentos da lagoa mirim de 6,81. Os pontos P1 e P3 possuíram uma menor granulometria e apresentaram índices de mais elevados quando comparados com a amostra P2. Estes dados são de extrema importância uma vez que estes parâmetros influenciam na sorção dos hidrocarbonetos na matriz (GU et al., 2017).

Tabela 8: Caracterização preliminar de sedimento: análise de matéria orgânica (\% \pm RSD\%), pH, umidade (\% \pm RSD\%).

\begin{tabular}{|l|l|l|l|}
\hline \multicolumn{1}{|c|}{ Ponto } & \multicolumn{1}{c|}{ Matéria Orgânica } & \multicolumn{1}{c|}{ pH } & \multicolumn{1}{c|}{ Umidade } \\
\hline P1 & $0,8 \pm 15,6$ & 6,9 & $24,8 \pm 2,1$ \\
\hline P2 & $0,5 \pm 23,3$ & 7,2 & $25,3 \pm 1,6$ \\
\hline P3 & $1,7 \pm 1,9$ & 6,4 & $27,4 \pm 1,6$ \\
\hline
\end{tabular}

RSD: desvio padrão relativo. 


\section{Aplicação da metodologia para amostras de sedimento}

A Tabela 9, apresenta os níveis de HPAS encontrados nas amostras analisadas bem como as razões diagnósticas. Pode-se verificar a presença de HPAs nos três pontos amostrais, predominando compostos de

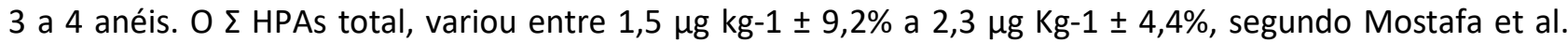
(2009), LHPAs < $100 \mu \mathrm{g}$ kg-1, indicam ambiente pouco impactado. Nenhum dos analitos apresentou valores acima dos níveis de efeito limiar (TEL) e a metodologia otimizada apresentou coeficientes de variação inferiores a $20 \%$ em acordo com os que foram encontradas por Xiang et al. (2018) em sedimento, água viva e corais feito que obteve resultados entre 6 a $22 \%$.

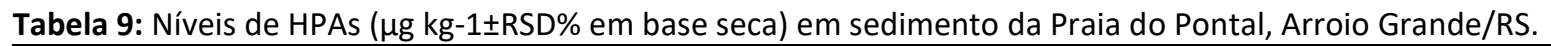

\begin{tabular}{|l|l|l|l|l|l|}
\hline HPAs & P1 & P2 & P3 & $\begin{array}{l}\text { TEL } \\
\mu \mathrm{kg}^{-1}\end{array}$ & $\begin{array}{l}\text { PEL } \\
\mu \mathrm{kg}^{-1}\end{array}$ \\
\hline Naftaleno & $\mathrm{nd}$ & $\mathrm{nd}$ & $\mathrm{nd}$ & 34,6 & 391,0 \\
\hline Acenaftileno & $\mathrm{nd}$ & $\mathrm{nd}$ & $\mathrm{nd}$ & 5,9 & 128,0 \\
\hline Acenafteno & $\mathrm{nd}$ & $\mathrm{nd}$ & $\mathrm{nd}$ & 6,7 & 88,9 \\
\hline Fluoreno & $0,6 \pm 2,7$ & $\mathrm{nd}$ & $1,2 \pm 3,9$ & 21,2 & 144,0 \\
\hline Fenantreno & $0,6 \pm 12,3$ & $0,5 \pm 4,3$ & $0,5 \pm 8,6$ & 41,9 & 515,0 \\
\hline Antraceno & $0,4 \pm 17,3$ & $0,1 \pm 15,4$ & $0,3 \pm 11,4$ & 46,9 & 245,0 \\
\hline Fluoranteno & $\mathrm{nd}$ & $0,2 \pm 0,2$ & $0,4 \pm 12,0$ & 111,0 & 2355,0 \\
\hline Pireno & $0,1 \pm 0,4$ & $0,2 \pm 0,2$ & $0,23 \pm 14,7$ & 53,0 & 875,0 \\
\hline Benzo(a)Antraceno & $0,12 \pm 12,8$ & $0,4 \pm 12,0$ & $\mathrm{nd}$ & 31,7 & 385,0 \\
\hline Criseno & $\mathrm{nd}$ & $0,1 \pm 5,9$ & $\mathrm{nd}$ & 57,1 & 862,0 \\
\hline Benzo(k)Fluoranteno & $\mathrm{nd}$ & $\mathrm{nd}$ & $\mathrm{nd}$ & - & - \\
\hline Benzo(b)Fluoranteno & $\mathrm{nd}$ & $\mathrm{nd}$ & $\mathrm{nd}$ & - & - \\
\hline Benzo(a)Pireno & $\mathrm{nd}$ & $\mathrm{nd}$ & $\mathrm{nd}$ & 31,9 & 782,0 \\
\hline Benzo(g,h,i)Perileno & $\mathrm{nd}$ & $\mathrm{nd}$ & $\mathrm{nd}$ & - & - \\
\hline Dibenzo(a,h)Antraceno & $\mathrm{nd}$ & $\mathrm{nd}$ & $\mathrm{nd}$ & 6,2 \\
\hline Indeno(1,2,3-c,d)Pireno & $\mathrm{nd}$ & $\mathrm{nd}$ & $\mathrm{nd}$ & 135,0 \\
\hline Total & $1,8 \pm 4,9$ & $1,5 \pm 9,2$ & $2,3 \pm 4,4$ & $979,8 *$ & - \\
\hline Fen/Ant & 1,4 & 5,4 & 2,3 & & - \\
\hline Ant/(Ant+Fen) & 0,4 & 0,2 & 0,3 & & \\
\hline Flu/Pir & - & 0,9 & 1,1 & & \\
\hline Flu/(Flu+Pir) & - & 0,5 & & \\
\hline
\end{tabular}

TEL= Nível limite de efeito; PEL= Nível de efeito provável. *CONAMA 454/2012.

Os resultados para as razões diagnósticas Fen/Ant, Ant/(Ant+Fen), Flu/(Flu + Pir) e Flu/Pir foram associadas a entradas pirogênicas. Os valores obtidos para Flu/(Flu+Pir) $>0,4$ e $<0,5$ são indicadores de combustão de combustíveis fósseis, enquanto Flu/(Flu+Pir) > 0,5 são de combustão de grama, madeira ou carvão (HASSAN et al., 2018).

A Tabela 10 apresenta os resultados para n-alcanos e os índices usados para determinar a origem dos n-alcanos no ambiente. De forma geral, foram encontrados n-alcanos entre C14 e C33 em todas as amostras acompanhados de mistura complexa não resolvida (MCNR), o $\Sigma$ n-alcanos variaram de 2,2 mg kg-1 \pm 9,5\% (P2), a 16,6 mg kg-1 \pm 4,0\% (P1). Estes resultados estão em acordo com as características físico químicas (matéria orgânica e granulometria) destes pontos. Neste estudo, as concentrações obtidas para n-alcanos estão abaixo dos resultados que foram encontrados na Colônia Z3 em Pelotas por Sanches Filho et al. (2013) que apresentou valores de 62,0 $\mu \mathrm{g}$ kg-1 $\pm 19,4 \%$ a 261,1 $\mu \mathrm{g}$ g-1 $\pm 15,0 \%$ e dos valores apresentados em diversos sedimentos obtidos na Ilha de Florianópolis por Assunção et al. (2018), que variaram de 18,0 $\mu \mathrm{g}$ g-1 $\pm 3,2 \%$ a 55,6 $\mu \mathrm{g} g-1 \pm 3,7 \%$, estes resultados confirmam que ambos ecossistemas estão sob intensa influência antropogênica, devido à proximidade de grandes cidades, diferente do que ocorre na área de estudo deste 
trabalho que está localizada em uma zona rural no Sul do Brasil. O desvio padrão relativo expresso em porcentagem que variou de 0,03 a 18,8\%, podem ser comparados com o estudo de hidrocarbonetos em sedimento, feito por Assunção et al. (2017), que obteve resultados entre 1,2 -11,1\%, com isto conclui-se que a precisão do método foi considerada aceitável.

Tabela 10: Níveis de $n$-alcanos (mg kg-1 \pm RSD\% em base seca) em sedimento da Praia do Pontal, Arroio Grande/RS.

\begin{tabular}{|c|c|c|c|}
\hline $\mathrm{N}$-alcanos & P1 & $\mathrm{P} 2$ & P3 \\
\hline Nonano & nd & nd & nd \\
\hline Decano & nd & nd & nd \\
\hline Undecano & nd & nd & nd \\
\hline Dodecano & nd & nd & nd \\
\hline Tridecano & nd & nd & nd \\
\hline Tetradecano & $0,7 \pm 0,8$ & $0,3 \pm 1,7$ & $0,7 \pm 4,4$ \\
\hline Pentadecano & $0,7 \pm 0,4$ & $0,3 \pm 1,6$ & $0,9 \pm 8,7$ \\
\hline Hexadecano & $2.0 \pm 0,5$ & $0,2 \pm 0,6$ & $1,8 \pm 1,9$ \\
\hline Heptadecano & $1,0 \pm 1,0$ & $0,8 \pm 7,2$ & $0,8 \pm 18,5$ \\
\hline Octadecano & $1,3 \pm 0,7$ & $0,5 \pm 7,0$ & $1,2 \pm 3,7$ \\
\hline Nonadecano & $0,8 \pm 0,7$ & $0,6 \pm 3,0$ & $0,8 \pm 15,3$ \\
\hline Eicosano & $1,1 \pm 0,4$ & $0,7 \pm 2,6$ & $1,2 \pm 8,4$ \\
\hline Heneicosano & $0,6 \pm 0,6$ & $0,4 \pm 9,4$ & $0,5 \pm 16,0$ \\
\hline Docosano & $0,8 \pm 0,3$ & $0,7 \pm 11,3$ & $0,7 \pm 15,7$ \\
\hline Tricosano & $0,7 \pm 0,03$ & $0,6 \pm 18,2$ & $0,5 \pm 14,2$ \\
\hline Tetracosano & $0,7 \pm 0,1$ & $0,6 \pm 14,4$ & $0,6 \pm 16,9$ \\
\hline Pentacosano & $0,7 \pm 0,6$ & $0,5 \pm 8,3$ & $0,5 \pm 14,1$ \\
\hline Hexacosano & $0,7 \pm 0,6$ & $0,8 \pm 14,7$ & $0,7 \pm 14,4$ \\
\hline Heptacosano & $0,7 \pm 0,1$ & $0,6 \pm 13,1$ & $0,7 \pm 16,1$ \\
\hline Octacosano & $0,8 \pm 0,1$ & $0,7 \pm 14,4$ & $0,6 \pm 17,5$ \\
\hline Nonacosano & $0,8 \pm 0,9$ & $0,6 \pm 7,9$ & $0,7 \pm 18,8$ \\
\hline Triacontano & $0,5 \pm 0,7$ & $0,5 \pm 5,3$ & $0,5 \pm 15,5$ \\
\hline Untriacontano & $0,7 \pm 0,03$ & $0,2 \pm 9,9$ & $0,6 \pm 16,7$ \\
\hline Dotriacontano & $0,6 \pm 0,8$ & $0,5 \pm 11,0$ & $0,6 \pm 16,3$ \\
\hline Tritriacontano & $0,6 \pm 0,6$ & $0,4 \pm 10,8$ & $0,5 \pm 18,9$ \\
\hline Tetratriacontano & nd & $0,4 \pm 8,1$ & nd \\
\hline Pentatricontano & nd & $\mathrm{d}$ & nd \\
\hline Hexatriacontano & nd & nd & nd \\
\hline Heptatriacontano & nd & nd & nd \\
\hline Octatriacontano & nd & nd & nd \\
\hline Nonatriacontano & nd & nd & nd \\
\hline Total & $16,6 \pm 4,0$ & $11,0 \pm 9,5$ & $14,8 \pm 11,1$ \\
\hline IPC & $1,8 \pm 0,9$ & $1,5 \pm 0,8$ & $1,8 \pm 0,9$ \\
\hline RTA & $0,8 \pm 0,4$ & $1,2 \pm 0,6$ & $0,8 \pm 0,4$ \\
\hline MCNR & $64,9 \pm 12,4$ & $76,6 \pm 12,6$ & $49,3 \pm 14,6$ \\
\hline MCNR/乏n-alcanos & $3,9 \pm 1,9$ & $7,0 \pm 3,5$ & $3,4 \pm 1,7$ \\
\hline
\end{tabular}

IPC: Índice Preferencial de Carbono; RTA: Razão Terrígeno Aquática; MCNR: Mistura Complexa não Resolvida.

Embora o $\Sigma \mathrm{n}$-alcanos indicam ambiente pouco impacto, a presença majoritária dos compostos $\mathrm{C} 16$, C18 e C20, indicam o aporte de resíduos de combustíveis fósseis. A razão MCNR/ $/$ n-alcanos que variou de 3,23 a 4,48, confirma uma contaminação recente por derramamento de óleos e combustíveis, principalmente no P1 e P3, Tabela 4 (TOLOSA et al., 2004; SANCHES FILHO et al., 2013). Neste estudo, os valores, do IPC foram menores que 2 (faixa de 1,8 a 1,5), os quais indicam insumos petrogênicos. A razão terrestre/aquática (RTA) apresentou valores que variaram de 1,2 a 0,8 (Tabela 5), indicando fontes aquáticas e terrígenas aos sedimentos (ASSUNÇÃO et al., 2018; BARBOSA et al., 2018).

\section{CONCLUSÕES}

A metodologia otimizada para a determinação de hidrocarbonetos, através da combinação de etapas de pré-sonificação juntamente com extração soxhlet, utilizando equipamentos disponíveis na maioria dos laboratórios de análise de contaminantes ambientais, apresentou-se satisfatória com resultados obtidos para 
limites de detecção, quantificação, precisão, exatidão e recuperação recomendados pelos órgãos ambientais. O procedimento resultou na redução do tempo de extração de 16 horas para 4 horas e 15 minutos e reduziu o volume de solventes utilizados de $300 \mathrm{~mL}$ para $100 \mathrm{~mL}$, quando comparados aos métodos oficiais EPA 3550C e EPA 3540C. A aplicação da metodologia nas amostras de sedimento da Praia do Pontal, demonstrou um ambiente pouco impactado, porem foi possível determinar as fontes de hidrocarbonetos relacionadas as atividades antrópicas. Desta forma, a metodologia otimizada constituiu em uma alternativa simples e eficiente, a ser utilizada em laboratórios para a caracterização e monitoramento destes contaminantes em sedimentos lagunares.

\section{REFERÊNCIAS}

APHA. Standard Methods for the Examination of Water and Wastewater. Washington, 2005.

ASSUNÇÃO, M. A.; FRENA, M.; SANTOS, A. P. S.; MADUREIRA, L. A. S.. Aliphatic and polycyclic aromatic hydrocarbons in surface sediments collected from mangroves with different levels of urbanization in southern Brazil. Marine pollution bulletin, v.119, n.1, p.439-445, 2017. DOI: http://doi.org/10.1016/i.marpolbul.2017.03.071

BARBOSA, J. C. S.; SANTOS, L. G.; SANT'ANNA, M. V.; SOUZA, M. R.; DAMASCENO, F. C.; ALEXANDRE, M. R.. Seasonal distribution of aliphatic hydrocarbons in the Vaza Barris estuarine system, Sergipe, Brazil. Marine pollution bulletin, v.104, n.1-2, p.343-346, 2016. DOI:

http://doi.org/10.1016/j.marpolbul.2016.01.037

BET, R.. Origem e destino da matéria orgânica sedimentar na região de desembocadura do Complexo Estuarino de Paranaguá-Paraná/Brasil utilizando marcadores orgânicos geoquímicos. Monografia (Faculdade de Oceanografia) Universidade Federal do Paraná, Pontal do Paraná, 2010.

BRITO, N. M.; JUNIOR, O. P. D. A.; POLESE, L.; RIBEIRO, M. L.. Validação de métodos analíticos: estratégia e discussão. Pesticidas: Revista de Ecotoxicologia e Meio Ambiente, v.13, p. 29-146, 2003. DOI:

http://dx.doi.org/10.5380/pes.v13i0

CAVALCANTE, R. M.; LIMA, D. M.; CORREIA, L. M.; NASCIMENTO, R. F.; SILVEIRA, E. R.; FREIRE, G. S.; VIANA, R. B.. Técnicas de extrações e procedimentos de clean-up para a determinação de hidrocarbonetos policíclicos aromáticos (HPA) em sedimentos da costa do Ceará. Química Nova, v.31, n.6, p.1371-1377, 2008. DOI:

http://dx.doi.org/10.1590/S0100-40422008000600019

COMMISSION REGULATION. N.836/2011: amending Regulation (EC) N.333/2007 laying down the methods of sampling and analysis for the official control of the levels of lead, cadmium, mercury, inorganic tin, 3-MCPD and benzo(a)pyrene in foodstuffs. 2011.

DJENNI, Z.; PINGRET, D.; MASON, T. J.; CHEMAT, F.. SonoSoxhlet: In situ ultrasound-assisted extraction of food products. Food analytical methods, v.6, n.4, p.1229-1233, 2013. DOI: http://doi.org/10.1007/s12161-012-9531-2
GU, Y. G.; LI, H. B.; LU, H. B.. Polycyclic aromatic hydrocarbons (PAHs) in surface sediments from the largest deep plateau lake in China: Occurrence, sources and biological risk. Ecological engineering, v.101, p.179-184, 2017. DOI: http://doi.org/10.1016/i.ecoleng.2017.02.007

HARTZELL, S. E.; UNGER, M. A.; VADAS, G. G.; YONKOS, L. T.. Evaluating porewater polycyclic aromatic hydrocarbonrelated toxicity at a contaminated sediment site using a spiked field-sediment approach. Environmental toxicology and chemistry, v.37, n.3, p.893-902, 2018. DOI: http://doi.org/10.1002/etc.4023

HASSAN, H. M.; CASTILLO, A. B.; YIGITERHAN, O.; ELOBAID, E. A.; AL-OBAIDLY, A.; AL-ANSARI, E.; OBBARD, J. P.. Baseline concentrations and distributions of Polycyclic Aromatic Hydrocarbons in surface sediments from the Qatar marine environment. Marine Pollution Bulletin, v.126, p.58-62, 2018. DOI: http://doi.org/10.1016/j.marpolbul.2017.10.093

INMETRO. Instituto Nacional de Metrologia, Normalização e Qualidade Industrial. Orientações sobre Validação de Métodos de Ensaios Químicos: DOQ-CGCRE-008. Brasília, 2003.

LOURENÇO, R. A.; MARTINS, C. C.; TANIGUCHI, S.; MAHIQUES, M. M.; MONTONE, R. C.; MAGALHÃES, C. A.; BÍCEGO, M. C.. Distribution and evolution of sterols and aliphatic hydrocarbons in dated marine sediment cores from the Cabo Frio upwelling region, SW Atlantic,

Brazil. Environmental Science and Pollution Research, v.24, n.24, p.19888-19901, 2017. DOI: http://doi.org/10.1007/s11356-017-9657-2

LUQUE-GARCIA, J. L.; CASTRO, M. L.. Ultrasound-assisted soxhlet extraction: an expeditive approach for solid sample treatment: application to the extraction of total fat from oleaginous seeds. Journal of Chromatography A, v.1034, n.1-2, p.237-242, 2004. DOI: http://doi.org/10.1016/j.chroma.2004.02.020

LUZ, L. P.; SANCHES FILHO, P. J.; DE SOUSA, E. E. H.; KERSTNER, T.; CARAMÃO, E. B.. Evaluation of surface sediment contamination by polycyclic aromatic hydrocarbons in colony Z3-(Patos Lagoon, Brazil). Microchemical Journal, v.96, n.1, p.161-166, 2010. DOI: http://doi.org/10.1016/j.microc.2010.03.003 
MOSTAFA, A. R.; WADE, T. L.; SWEET, S. T.; AL-ALIMI, A. K. A.; BARAKAT, A. O.. Distribution and characteristics of polycyclic aromatic hydrocarbons (PAHs) in sediments of Hadhramout coastal area, Gulf of Aden, Yemen. Journal of Marine Systems, v.78, n.1, p.1-8, 2009. DOI: http://doi.org/10.1016/j.jmarsys.2009.02.002

\section{MOZETO, A. A.. Sedimentos e particulados lacustres:} amostragens e análises biogeoquímicas. Amostragem em Limnologia. São Carlos: RiMa, 2004.

OLIVEIRA, H. A. D.; FERNANDES, E. H. L.; MÖLLER JUNIOR, O. O.; COLLARES, G. L.. Processos hidrológicos e hidrodinâmicos da Lagoa Mirim. Revista Brasileira de Recursos Hídricos, v.20, n.1, p.34-45, 2015. DOI: http://doi.org/10.21168/rbrh.v20n1.p34-45

PENA, M. T.; CASAIS, M. C.; MEJUTO, M. C.; CELA, R.. Development of a matrix solid-phase dispersion method for the determination of polycyclic aromatic hydrocarbons in sewage sludge samples. Analytica Chimica Acta, v.626, n.2, p.155-165, 2008. DOI: http://doi.org/10.1016/i.aca.2008.07.053

QU, C.; LI, J.; ALBANESE, S.; LIMA, A.; WANG, M.; SACCHI, M.; VIVO, B.. Polycyclic aromatic hydrocarbons in the sediments of the Gulfs of Naples and Salerno, Southern Italy: Status, sources and ecological risk. Ecotoxicology and environmental safety, v.161, p.156-163, 2018. DOI: http://doi.org/10.1016/i.ecoenv.2018.05.077

SANCHES FILHO, P. J.; LUZ, L. P. D.; BETEMPS, G. R.; SILVA, M. D. R. G. D.; CARAMÃO, E. B.. Studies of n-alkanes in the sediments of colony Z3 (Pelotas-RS-Brazil). Brazilian Journal of Aquatic Science and Technology, v.17, n.1, p.27-33, 2013. DOI: http://doi.org/10.14210/bjast

SANCHES FILHO, P. J.; BÖHM, E. M.; BÖHM, G. M.; MONTENEGRO, G. O.; SILVEIRA, L. A.; BETEMPS, G. R. Determination of hydrocarbons transported by urban runoff in sediments of São Gonçalo Channel (Pelotas-RS, Brazil). Marine pollution bulletin, v.114, n.2, p.1088-1095, 2017. DOI: http://doi.org/10.1016/j.marpolbul.2016.10.024

SANTOS, I. R.; BAISCH, P.; LIMA, G. T. N. P.; SILVA FILHO, E. V.. Metais pesados em sedimentos superficiais da Lagoa Mirim, fronteira Brasil-Uruguai. Geochimica Brasiliensis, v.17, n.1, 2003. DOI: http://doi.org/10.21715/gb.v17i1.196

SOUZA, M. R.; SANTOS, E.; SUZARTE, J. S.; CARMO, L. O.; FRENA, M.; DAMASCENO, F. C.; ALEXANDRE, M. R.. Concentration, distribution and source apportionment of polycyclic aromatic hydrocarbons (PAH) in Poxim River sediments, Brazil. Marine Pollution Bulletin, v.127, p.478-

483, 2018. DOI:

http://doi.org/10.1016/i.marpolbul.2017.12.045

SUGUIO, K.. Introdução à sedimentologia. São Paulo: Edgard Blücher, 1973.

SUN, R.; SUN, Y.; LI, Q. X.; ZHENG, X.; LUO, X.; MAI, B.. Polycyclic aromatic hydrocarbons in sediments and marine organisms: Implications of anthropogenic effects on the coastal environment. Science of the Total Environment v.640, p.264-272, 2018. DOI: http://doi.org/10.1016/j.scitotenv.2018.05.320

TEÓFILO, R. F.; FERREIRA, M.. Quimiometria II: planilhas eletrônicas para cálculos de planejamentos experimentais, um tutorial. Química Nova, v.29, n.2, p.338-350, 2006.

TOLOSA, I.: MORA, S.; SHEIKHOLESLAMI, M. R.; VILLENEUVE, J. P.; BARTOCCI, J.; CATTINI, C.. Aliphatic and aromatic hydrocarbons in coastal Caspian Sea sediments. Marine Pollution Bulletin, v.48, p.44-60, 2004. DOI: https://doi.org/10.1016/S0025-326X(03)00255-8.

UNEP. United Nations Environment Programme.

Determination of petroleum hydrocarbons in sediments.

References Methods for Marine Pollution studies. 1992.

USEPA. United States Environmental Protection Agency. Method 3540C: Soxhlet Extraction. Washington: US Environmental Protection Agency, 1996.

USEPA. United States Environmental Protection Agency. Method 5035A: Closed-System Purge-And-Trap and Extraction for Volatile Organics in Soil and Waste Samples. Washington: US Environmental Protection Agency, 2002.

USEPA. United States Environmental Protection Agency. Method 3550C-Ultrasonic Extraction. Washington: US Environmental Protection Agency, 2007.

XIANG, N.; JIANG, C.; YANG, T.; LI, P.; WANG, H.; XIE, Y.; DIAO, X.. Occurrence and distribution of polycyclic aromatic hydrocarbons (PAHs) in seawater, sediments and corals from Hainan Island, China. Ecotoxicology and environmental safety, v.152, p.8-15, 2018. DOI: http://doi.org/10.1016/j.ecoenv.2018.01.006

YOON, J.; CAO, X.; ZHOU, Q.; MA, L. Q.. Accumulation of Pb, $\mathrm{Cu}$, and $\mathrm{Zn}$ in native plants growing on a contaminated Florida site. Science of the total environment, v.368, n.2-3, p.456-464, 2006. DOI: http://doi.org/10.1016/i.scitotenv.2006.01.016

A CBPC - Companhia Brasileira de Produção Científica (CNPJ: 11.221.422/0001-03) detém os direitos materiais desta publicação. Os direitos referem-se à publicação do trabalho em qualquer parte do mundo, incluindo os direitos às renovações, expansões e disseminações da contribuição, bem como outros direitos subsidiários. Todos os trabalhos publicados eletronicamente poderão posteriormente ser publicados em coletâneas impressas sob coordenação da Sustenere Publishing, da Companhia Brasileira de Produção Científica e seus parceiros autorizados. Os (as) autores (as) preservam os direitos autorais, mas não têm permissão para a publicação da contribuição em outro meio, impresso ou digital, em português ou em tradução. 